\title{
APPROACHES TO MESSENGER RNA DETECTION - COMPARISON OF METHODS
}

\author{
Zdeněk Dvořák ${ }^{\mathrm{a} *}$, Jean-Marc Pascussi ${ }^{\mathrm{b}}$, Martin Modrianskýa
}

\author{
a Institute of Medical Chemistry and Biochemistry, Faculty of Medicine, Palacký University Olomouc, Hněvotínská 3, \\ 77515 Olomouc, Czech Republic. \\ b CNRS - INSERM - U128, 1919 Route de Mende, 34293 Montpellier, France.
}

Received: September 23, 2003; Accepted: October 15, 2003

Key words: Messenger RNA / Northern blot / Ribonuclease protection assay / Real time polymerase chain reaction

Detection of messenger RNA is an important part of current biomedical research, although utilized for decades. This communication endeavors to compare three most commonly used techniques of mRNA detection, i.e. Northern blot, ribonuclease protection assay (RPA), and real-time polymerase chain reaction (RT-PCR). Principles and general procedures of these methods are described, and advantages and weaknesses of each are discussed in terms of their specificity, sensitivity, difficulty, time and material demands as well as health and environmental risks. We conclude that choice of any method discussed depends on particular purpose, experience of the researcher, and on laboratory equipment and organization.

\section{INTRODUCTION}

Messenger ribonucleic acid (mRNA) is an essential functional constituent of a DNA-containing cell in any living organism. It is a polymer of four nucleotides, the order of which gives primary structure unique to an individual functional gene or a pseudogene. During the process of gene expression, the functional single strand structure of mRNA is synthesized and serves thereafter as an intermediate template for translation process in proteosynthesis. In many fields of current research, including molecular biology, toxicology, physiology, pharmacology, biochemistry etc., the detection of individual mRNAs is an indispensable tool. From the point of view of analytical chemistry, the detection/determination of mRNA represents the following: 1) The nature of mRNA as a macromolecule; 2) Identical physico-chemical properties of mRNAs representing different genes; 3) Very low level/concentration of mRNA of interest; 4) Low stability at room temperature together with extreme sensitivity to destructive action of ribonucleases and $\mathrm{pH}$ change in aqueous milieu. Taken together, any of methods employed for mRNA detection face the problems of sensitivity, specificity, and stability.

Hereby we attempt to describe in brief and to compare the three most commonly used techniques of mRNA detection, i.e. Northern blot (NB), ribonuclease protection assay (RPA) and real-time polymerase chain reaction (RT-PCR), with respect to the abovementioned features of mRNA analysis. Each technique is first described and than its advantages and weak spots are emphasised. For the "rating" of the methods, we used literature data as well as our own experimental experience. We chose an approach similar to "SWOT" analysis (Strength vs Weakness and Opportunity vs Threats) in economy.
We do not claim that the presented discourse is exhaustive, it is intended to be rather a simplified guide for researchers starting their work in the mRNA field.

\section{SAMPLE PREPARATION}

mRNA obviously comes from biological samples, i.e. tissues or cells, consisting of vast number of various micro- and macromolecules, it is therefore primordial to isolate it properly. The widespread technique used for years employs commercial reagent Trizol ${ }^{\circledR}$. The principle is based on cell/tissue lysis by this reagent, followed by extraction (phenol/chloroform/water), precipitation (isopropyl alcohol), and washing (75\% ethanol) steps ${ }^{1}$. Resulting pellet of RNA is then dissolved either in formamide (for NB and RPA) or water (for RT-PCR). Note that using this method the total RNA is obtained, i.e. a mixture of all mRNA, tRNA, and rRNA. One must bear in mind that mRNA represents only about $1 \%$ of that total amount. Depending on a particular case, the yield is around $50-250 \mu \mathrm{g}$ of total RNA per 10 millions of mammalian cells. Advantage of Trizol ${ }^{\circledR}$ technique is the possibility to obtain genomic DNA as well as total cellular protein from the same sample by an extended extraction procedure.

It is possible, and in case of RT-PCR highly recommended, to isolate mRNA directly from tissue homogenate, cell culture, or total RNA. Commercial kits for this purpose are available which utilize adsorption of mRNA to sorbents with anchored oligo(dT) probe due to interaction with the poly-A end of mRNAs ${ }^{2}$. Following extensive washing the mRNA is eluted and used for further applications. Advantage is the purity of obtained mRNA. This, 
however, is accompanied by a higher risk of ribonuclease contamination.

Since RNA is extremely sensitive to the action of ribonucleases, it is essential to use disposable laboratory ware (eppendorfs, tubes, tips) autoclaved before use. Plastic gloves are strictly recommended for working with RNA and it is useful and convenient to designate "RNAse free zone" in the laboratory. Following isolation, the concentration of RNA in samples is determined by $\mathrm{UV}$-spectrometry at $260 \mathrm{~nm}$. It is recommended to evaluate absorbance at $280 \mathrm{~nm}$ as well to have an estimate of proteins impurities, with the ratio $\mathrm{A}_{260} / \mathrm{A}_{280}>1.8$ being satisfactory for further use of a particular sample. High absorbance at $\lambda<260 \mathrm{~nm}$ (absence of a clear peak at $260 \mathrm{~nm}$ ) suggests traces of phenol contaminating the sample. Residual phenol may be removed by an additional wash step and it is needed particularly for RT-PCR. Owing to thermal instability of mRNA, the samples are stored (in formamide; FA or water; $\mathrm{W}$ ) in $-80{ }^{\circ} \mathrm{C}$ freezer and on ice when in use.

\section{NORTHERN BLOT}

Essentially a variation of a method developed for DNA detection, it serves two main purposes: i) detection of mRNA presence in a cell as evidence for gene transcription, and ii) estimation of mRNA size. Because of lower sensitivity, it is limited to high abundance mRNA. On the other hand, shift from low to high abundance, i.e. inducibility, of a gene can be easily demonstrated because of this. Different size mRNAs of a specific gene detected using this technique suggest presence of a pseudogene or a splice variant which can be utilized gene expression regulation studies.

Method description: Samples dissolved in either FA or W can be analysed by this, let us say, classic technique ${ }^{3}$. Depending on the gene of interest expression level an aliquot of $10-50 \mu \mathrm{g}$ of total RNA is mixed with a formaldehyde-based loading buffer and denatured by heat. Then, electrophoretic separation of RNAs is performed on an agarose gel under denaturing conditions. Formaldehyde is present both in the gel and the migration buffer ensuring the inhibition of ribonuclease activity. Formaldehyde can be substituted by other substances, e.g. glyoxal, but with a bit higher risk of ribonuclease contamination. Procedure is performed in a horizontal arrangement, with constant stirring of the migration buffer and using constant current. The time of analysis varies between 1-2 $\mathrm{h}$ and 5-6 h for small and large gels, respectively. Separation is followed by transfer of RNAs onto the nylon membrane, driven by capillary forces, the so-called Northern blot. The procedure usually runs overnight. Quality of transfer is quickly checked under UV light (254 nm) when two major bands of ribosomal $18 \mathrm{~S}$ and $28 \mathrm{~S}$ RNA fraction are visible. According to the time schedule/equipment, the membrane is: a) exposed to an intense UV light to induce mRNA cross-link (for immediate use); or b) dried under vacuum. Following the latter procedure, the membrane can be stored in plastic wrap for months.

Since mRNA (+, sense) is being synthesised by transcription of a template DNA strand, it has the nucleotide sequence of the coding DNA strand (note that uridine is present instead of thymidine). Accordingly, an appropriate plasmid with the cloned cDNA fragment of desirable gene is used for the synthesis of the probe, which has then sequence of the original template DNA strand. Commercial kits are used, employing DNA-dependent DNA polymerase (Klenow) and microcolumns for probe purification. The probe is most often radio-labelled using ${ }^{32} \mathrm{P}-\alpha-\mathrm{dCTP}$ or by biotin. The principle of detection is the hybridization of mRNA fixed in membrane with the labelled cDNA probe (sequence complementary to the mRNA) forming a double stranded RNA-DNA structure. Hybridization is usually carried out overnight in a rotating oven and detected by: a) autoradiography or Phosphoimager apparatus for ${ }^{32} \mathrm{P}$ labelled probe; or b) immuno-chemiluminiscence detection for biotinylated probe.

A representative example of NB is shown in Fig 1.

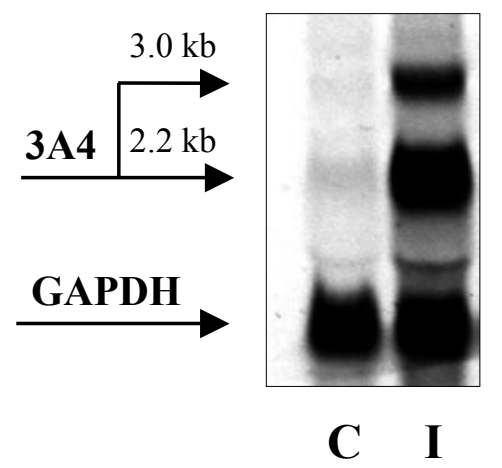

Fig. 1. Example of Northern blot analysis. Northern blot of total RNA $(20 \mu \mathrm{g})$ obtained from primary human hepatocytes using Trizol isolation procedure. $\mathrm{kb}=$ = kilobase; GAPDH = glyceraldehyde-3-phosphate dehydrogenase; $3 \mathrm{~A} 4$ = cytochrome $\mathrm{P} 450$ isoform $3 \mathrm{~A} 4$ (band at $2.2 \mathrm{~kb}$ ); $\mathrm{C}=$ control cells; $\mathrm{I}=$ cells treated with $3 \mathrm{~A} 4$ inducer rifampicin. Band at $3.0 \mathrm{~kb}$ corresponds to $3 \mathrm{~A} 4$ pseudogene.

SWOT: Strength of the method is its simplicity. Time schedule allows spreading of the work into two stages of sample-separation and hybridization-detection possibly performed weeks apart. Specificity is relatively high. Financial demands are the lowest of all described methods with added cost if Phosphoimager apparatus is being used. Weakness is linked to the work and environmental risks when high doses of radioactivity (on the order of $10^{7} \mathrm{cpm}$ ) and formaldehyde are used. The sensitivity of Northern blot technique is the lowest of all the described methods. Opportunity emerges in the possibility to detect two or more genes in one hybridization step (with respect to 
separation efficiency and properties of the probe) and/or to perform additional detection after former RNA-DNA complex deshybridization. Again the time schedule of the method brings the chance to optimise the work or to transport the samples which is frequently important for co-operation between laboratories. Threat is represented by the risk of mRNA degradation during electrophoresis ( $\mathrm{pH}$ change).

\section{RIBONUCLEASE PROTECTION ASSAY}

This method can be used for detection and, to some extent, to quantification of mRNA. Its full potential, however, lies in the possibility to map RNA termini, to determine alternative splicing and even single base mutations. Positions of introns within a corresponding gene can be determined as well.

Method description: In this case, mRNA complementary to the detected, i.e. antisense (-) mRNA, serves as the probe ${ }^{4,5}$. In contrast to the NB principle, probe preparation and hybridisation precedes the separation step. A sequence of usually 200-450 bp corresponding to the desired mRNA is cloned into a plasmid between SP6, T7 or T3 promoter and a stop codon. For ribo-probe preparation, the plasmid is linearised such that it can be transcribed in the $3^{\prime}-5$ ' direction, contrary to the complementary strand which is transcribed in the 5'-3' direction. Then using corresponding DNA-dependent RNA polymerase (SP6, T7, T3), mixture of nucleotide triphosphates (ATP, GTP, TTP, UTP) and radioactively labelled ${ }^{32} \mathrm{P}-\alpha-\mathrm{UTP}$, the radio-labelled probe is syntesised as the anti-sense (-) mRNA. When the original plasmid is linearised to allow the correct transcription, the sense (+) mRNA identical to the detected one is synthesised and can serve as a standard (positive control). Following the transcription, the radio-labelled riboprobe is sequentially purified by 1) extraction/precipitation (phenol/ chloroform reagent); 2) electrophoresis on acrylamide gel containing urea; 3 ) extraction from acrylamide gel and precipitation. A riboprobe is then dissolved in formamide and stored in $-20{ }^{\circ} \mathrm{C}$. Presence of ${ }^{32} \mathrm{P}$ is limiting factor for storage because of half-life of approx. 15 days. As an alternative to radiolabeling, it is possible to use nucleotides labeled with e.g. biotin or digoxigenin, usually followed by indirect detection by chemiluminescence or fluorescence. However, nucleotides labeled in this fashion are not efficiently used by RNA polymerases necessary for riboprobe synthesis hence lowering the probe yield. Sensitivity of detection may also be lower than in case of radiolabeled probes making detection of low abundance mRNA difficult if not impossible.

Analytic procedure begins by hybridization, usually carried out overnight, when aliquot of 10-50 $\mu \mathrm{g}$ of total RNA in FA is incubated with riboprobe, and a double strand mRNA structure is formed. Mixture is then incubated with ribonucleases $\mathrm{A}$ and $\mathrm{T} 1$ which attack single strand RNA only. Double strand RNA structures are protected - hence the name of the method "ribonuclease protection assay". Since RNAses digest RNA even if a single base pair mismatch is present, the method provides almost absolute specificity. In parallel, riboprobe itself is treated to reveal the quality of RNA digestion (negative control, digested probe). Following RNA digestion the RNAses are eliminated by protease K cleavage. Remaining double strand RNA is extracted by phenol/chloroform, precipitated, and dissolved in a loading buffer based on FA. Prior to loading on a gel, double strand structure of RNA is disrupted by heat. Electrophoretic separation is performed in vertical arrangement on polyacrylamide gel containing urea. Constant current is applied and the time of analysis is between 1.5-2.5 hours. The gel is then soaked in a fixation solution and dried under vacuum. Detection, as for NB, is performed by autoradiography or Phosphoimager apparatus.

Representative example of RPA is shown in Fig 2.

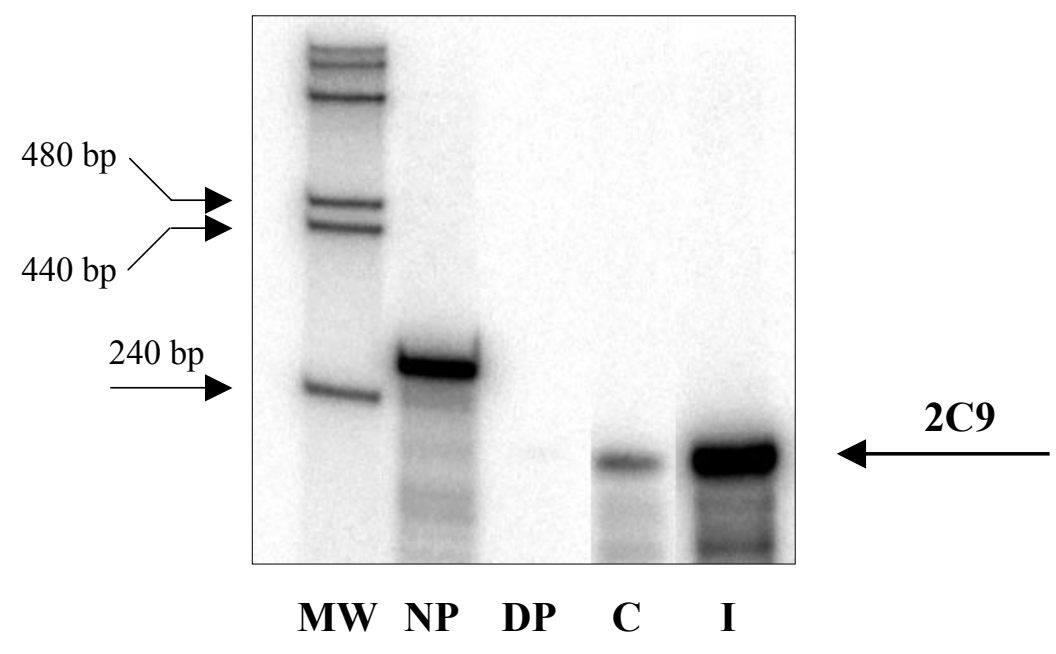

Fig. 2. Example of Ribonuclease protection assay analysis. RPA of total RNA ( $20 \mu \mathrm{g})$ isolated from primary human hepatocytes using Trizol isolation procedure. $\mathrm{bp}=$ base pair; $2 \mathrm{C} 9$ = cytochrome $\mathrm{P} 450$ isoform 2C9; $\mathrm{MW}=$ molecular weight marker; $\mathrm{NP}=$ native probe; $\mathrm{DP}=$ digested probe; $\mathrm{C}=$ control cells; $\mathrm{I}=$ cells treated with $2 \mathrm{C} 9$ inducer rifampicin. 
SWOT: Strength: Owing to its unique principle, the method gives nearly absolute specificity. It is therefore the most specific from the three methods under review ${ }^{6}$. Consequently, the design of the probe is intended to recognize a specific sequence like in other techniques. Compared to the NB, RPA is approximately ten times more sensitive. Weakness: The method is time consuming and requires a highly skilled operator. The work organisation is very tiresome for the operator because there is no chance for postponing any of the steps after hybridization. Furthermore, economical expenses are higher as compared to NB. Opportunity: Due to its high specificity, two or several genes with high homology in primary structure can be distinguished. Recall again that only one base pair mismatch leads to the cleavage by RNAses. Threat: Since the procedure consists of many steps, the risk of RNA degradation in sample during the analysis is very high and occurs quite frequently. The risk of radioactivity contamination when handling samples and particularly during riboprobe preparation is also considerable, even though the activities used are much lower than in the NB method.

\section{REAL TIME POLYMERASE CHAIN REACTION}

Thanks to its sensitivity even very low abundance mRNA can be detected using this technique. Other frequent uses include: primary validation of gene expression and hence confirmation of microarray data, splice variant analysis, allelic discrimination, viral titre (e.g. HCV), bacterial species identification, and single nucleotide polymorphism genotyping.

Method description: This method takes an advantage from well known polymerase chain reaction (PCR) which is used for DNA detection? ${ }^{7}$ The abbreviation RT-

A

\begin{tabular}{|c|c|c|}
\hline RIF $(\mu \mathrm{M})$ & Actin & CYP3A4 \\
\hline 0 & 14.69 & 27.84 \\
\hline 1 & 14.86 & 22.94 \\
\hline 5 & 15.07 & 22.24 \\
\hline 10 & 14.78 & 21.56 \\
\hline 20 & 15.31 & 21.52 \\
\hline
\end{tabular}

is somewhat ambiguous standing on one hand for reverse transcription reaction, a key step in the procedure, and on the other hand for real time format of the analysis when Lightcycler apparatus is used ${ }^{8}$.

First of all, the reverse transcription reaction is performed using RNA-dependent DNA polymerase (reverse transcriptase), synthesising the cDNAs complementary to the mRNAs present in the sample. It is sine qua non to use sample of RNA in water free of organic solvents residues, e.g. phenol, isopropanol, chloroform, ethanol etc., to avoid inhibition of reverse transcriptase. For the starting material it is possible to use total RNA, at least $1 \mu \mathrm{g}$, but isolated mRNA is more appropriate giving higher probability of low copy mRNA transcription. The synthesised cDNA is mixed with DNA-dependent DNA polymerase, sufficient amount of deoxynucleotide triphosphates (dATP, dCTP, dGTP, and dTTP), and fluorescently labeled oligonucleotide primers. As in the case of "classical" PCR, one cycle of a PCR reaction consists of strand separation, annealing, and elongation steps. Duration of and temperature during each of the three steps are set on the thermocycler apparatus in use. Fluorescence is monitored in each sample following every cycle of the PCR reaction. When threshold fluorescence intensity is reached due to increased number of copies of the cDNA of interest, the fluorescence linearly increases in each subsequent cycle eventually reaching a maximum plateau. The threshold is so-called "crossing point" which is essential for comparison between samples. Computer software provided with the Lightcycler usually calculates the cycle number corresponding to the crossing point using second derivative maximum. If calibrated for known number of cDNA copies present in a sample, RT-PCR can be used for very precise quantification of original mRNA copies per number of cells ${ }^{8,9}$. Representative computer output from a Lightcycler apparatus is shown in Fig 3.

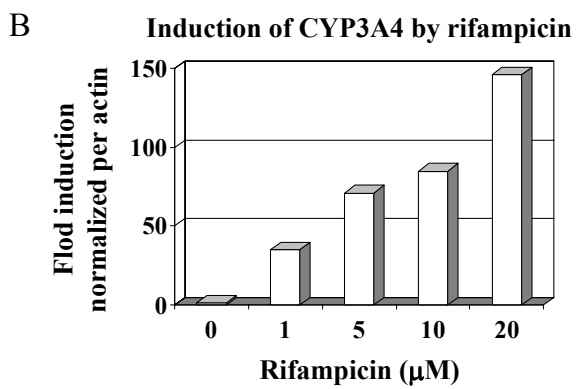

Fig. 3. Example of Real-Time Polymerase Chain Reaction analysis. RT-PCR computer data from total RNA isolated from primary human hepatocytes using Trizol procedure. Panel A) shows cycle thresholds for CYP3A4 and actin mRNA. Panel B) shows fold induction of CYP3A4 gene by rifampicin. Data are normalized per actin value. 
SWOT: Strength: Method is elegant, safe, simple to use, and requires only minimum experience and skills. It provides maximum sensitivity of all three discussed methods (approx. 10000 times more sensitive than NB) and among them it is the only one allowing quantification. Weakness: This method is the most expensive: Lightcycler apparatus, fluorescently labeled primers, and mRNA isolation kits are the principal costs. There is a need of highly pure samples to be used, free of organic solvents, often present after Trizol isolation increasing the need for alternative isolation procedures. Opportunity: Owing to its high sensitivity, a large number of genes can be analysed from a single mRNA preparation. Threat: The selection and design of the primers must be done carefully because of the possibility, albeit minuscule, that other homologous genes may be detected. The risk of RNA degradation, until the reverse transcription step is performed, is high because of water used as solvent. Contamination of RNA by fragments of genomic DNA can occur if Trizol is used for extraction. Consequently, it cannot be distinguished whether genomic DNA or reverse transcript are detected.

\section{CONCLUSION}

With the human genome now available a principal question was raised: what do all those genes do? In view of many a researcher this question translates primarily into the problem of: Are all those genes transcribed? It means to look for mRNA occurrence within a cell as many of the genes may be cell type specific. Area of genomics is not the only one with craving for mRNA data. The three methods described herein are the tools currently available for a researcher to deal with a particular problem involving mRNA.

We offer a SWOT analysis in attempt to help others during pros and cons consideration when selecting a method for their purpose. Things to consider are obviously intertwined as specificity of each method is linked to financial demands together with health and environmental risks. An ideal of a highly specific, safe, and inexpensive method for mRNA detection does not exist. Quite often combination of two, out of the three methods discussed, is utilized in many a lab throughout the world. This is important especially in pilot studies in order to confirm data obtained. Therefore we recommend to have one of these methods for routine use as the core method and then to select another one not as an alternative but as a confirmation tool.

\section{ACKNOWLEDGEMENT}

The authors gratefully acknowledge financial support from the Ministry of Education of the Czech Republic grant MSM 151100003.

\section{REFERENCES}

1. Chomczynski P, Sacchi N. (1993) Single-step method of RNA isolation by acid guanidinium thiocyanate-phenol-chloroform extraction. Anal Biochem 162, 156-9.

2. Kingston RE. (1993) Preparation of Poly(A) ${ }^{+}$RNA. In: Current Protocols in Molecular Biology (Eds.: Ausubel EM, Brent R, Kingston RE, Moore DD, Siedman JG, Smith JA, Struhl K), p 4.5.1.-4.5.3. John Wiley and Sons, New York, NY, 1993.

3. Brown T. (1993) Analysis of RNA by Northern and Slot Blot Hybridization. In: Current Protocols in Molecular Biology (Eds.: Ausubel EM, Brent R, Kingston RE, Moore DD, Siedman JG, Smith JA, Struhl K), p 4.9.1.-4.9.14. John Wiley and Sons, New York, NY, 1993.

4. Gilman M. (1993) Ribonuclease protection assay. In: Current Protocols in Molecular Biology (Eds.: Ausubel EM, Brent R, Kingston RE, Moore DD, Siedman JG, Smith JA, Struhl K), p 4.7.1.-4.7.8. John Wiley and Sons, New York, NY, 1993.

5. Melton DA, Krieg PA, Rebagliati MR, Maniatis T, Zinn K, Green MR. (1984) Efficient in vitro synthesis of biologically active RNA and RNA hybridization probes from plasmids containing a bacteriophage SP6 promoter. Nucleic Acids Res 12, 7035-56.

6. Rottman JB. (2002) The ribonuclease protection assay: a powerful tool for the veterinary pathologist. Vet Pathol 39, 2-9.

7. Erlich HA. (ed.) (1989) PCR Technology: Principles and Applications for DNA amplification. Stockton Press, London.

8. Wilkening S, Bader A. (2003) Influence of culture time on the expression of drug-metabolizing enzymes in primary human hepatocytes and hepatoma cell line HepG2. J Biochem Mol Toxicol 17, 207-13.

9. Wilkening S, Stahl F, Bader A. (2003) Comparison of primary human hepatocytes and hepatoma cell line HepG2 with regard to their biotransformation properties. Drug Metab Dispos 31, $1035-42$. 